\title{
Interpreting Supersymmetry
}

\author{
David John Baker \\ Department of Philosophy, University of Michigan \\ djbaker@umich.edu
}

October 7, 2018

\begin{abstract}
Supersymmetry in quantum physics is a mathematically simple phenomenon that raises deep foundational questions. To motivate these questions, I present a toy model, the supersymmetric harmonic oscillator, and its superspace representation, which adds extra anticommuting dimensions to spacetime. I then explain and comment on three foundational questions about this superspace formalism: whether superspace is a substance, whether it should count as spatiotemporal, and whether it is a necessary postulate if one wants to use the theory to unify bosons and fermions.
\end{abstract}

\section{Introduction}

Supersymmetry-the hypothesis that the laws of physics exhibit a symmetry that transforms bosons into fermions and vice versa-is a long-standing staple of many popular (but unconfirmed) theories in particle physics. This includes several attempts to extend the standard model as well as many research programs in quantum gravity, such as the failed supergravity program and the still-ascendant string theory program.

Its popularity aside, supersymmetry (SUSY for short) is also a foundationally interesting hypothesis on face. The fundamental equivalence it posits between bosons and fermions is prima facie puzzling, given the very different physical behavior of these two types of particle. And supersymmetry is most naturally represented in a formalism (called superspace) that modifies ordinary spacetime by adding Grassmann-valued anticommuting coordinates. It 
isn't obvious how literally we should interpret these extra "spatial" dimensions. ${ }^{1}$ So supersymmetry presents us with at least two highly novel interpretive puzzles.

Only two philosophers of science have taken up these questions thus far. In one of the earliest philosophical essays directed at the interpretation of string theory, Robert Weingard (1988) commented briefly on both questions. More recently Tushar Menon (forthcoming) has begun to work on the question of how literally to understand the superspace formalism. This is not a large literature, and despite its depth and sophistication, Menon's work should hardly be the last word on the topic. There is considerable room for further debate.

Moreover, supersymmetry is not an outrageously challenging topic. Its essential features can be understood through simple models derived from one of the most basic quantum mechanical systems, the simple harmonic oscillator. Using this model to bring the reader quickly up to speed (which will be the task of Section 2), I hope to provoke more philosophers to turn their attention to SUSY and the question of the interpretation of superspace.

With the supersymmetric oscillator model on the table, I'll raise (in Section 3) three closely-connected interpretive questions about SUSY. First is an ontological question: what is the most fundamental geometric structure posited by SUSY theories? In particular, if a SUSY theory is one day confirmed by experiment, should we adopt substantivalism about superspace, in keeping with the common opinion that spacetime theories license substantivalism about their most basic spatiotemporal structure? This will be the topic of Section 3.1.

A second question, which is closely related but not quite the same: is superspace the spacetime background of SUSY theories, or are these theories best understood as existing in ordinary relativistic spacetime? As Menon has pointed out, this question is closely related to the recent work of Eleanor Knox (2014) on functionalism about spacetime. Menon has argued that in light of (his preferred modification of) Knox's approach, we should conclude that superspace is (in a particular sense to be explained below) the spacetime in which SUSY theories exist. This conclusion may be correct-but I will show (in Section 3.2) that Menon's argument is not quite sufficient to prove it, and that the answer may depend on whether substantivalism about superspace is justified.

Finally, in his seminal essay on string theory, Weingard suggests that an important

\footnotetext{
${ }^{1}$ As we will see, it isn't obvious that the term "spatial" should apply to these coordinates, but they are certainly not temporal.
} 
explanatory issue hinges on these interpretive questions about superspace. It is tempting to think that SUSY achieves a sort of ontological unification, by representing bosons and fermions as fundamentally the same sort of entity. Weingard argues that this unification is only possible, however, if we understand superspace as spacetime. In Section 3.3, I will show that Weingard's argument rests on a mistaken assumption about the difference between spacetime symmetries and internal symmetries.

But let's not get ahead of ourselves. Before we get to the philosophy, we'd better take a look at the physics.

\section{The supersymmetric harmonic oscillator}

SUSY is normally thought of as a symmetry of quantum field theories (QFTs) or string theories, because these are the supersymmetric theories believed to have physical applications. But SUSY can also be defined on much simpler models of ordinary quantum mechanics. We will focus here on the supersymmetric version of the well-known quantum simple harmonic oscillator. Due to the often-noted analogy between harmonic oscillators and free quantum fields, this model will turn out to be analogous to supersymmetric quantum field theories in most foundationally relevant respects.

I'll proceed by presenting two separate non-supersymmetric models, the bosonic oscillator and the fermionic oscillator, and explaining their similarities to bosonic and fermionic quantum field theories. (In brief, a harmonic oscillator is formally identical to a free QFT on 0+1-dimensional spacetime.) Then I'll show how to combine these into a single model representing a supersymmetric oscillator. Finally I will construct the superspace version of this SUSY oscillator, which is a quantum field theory on superspace. ${ }^{2}$

The most familiar part of this whole apparatus will be the bosonic oscillator. This is just the ordinary simple harmonic oscillator (in one spatial dimension) covered in introductory quantum mechanics textbooks. Its lowest-energy state is the ground state $|0\rangle$. The higher eigenstates of energy $|1\rangle,|2\rangle$, etc then correspond to the first, second, etc, modes of vibration of the oscillator, and its energy levels are given by the expectation values of the Hamiltonian (energy operator) $H_{B}$ in these states. For any eigenstate $|n\rangle$, this expectation value $\left\langle n\left|H_{B}\right| n\right\rangle$ is given by the ground state energy plus some constant times $n$.

\footnotetext{
${ }^{2}$ My presentation of this model is based off of Susskind (2012) and Bagchi (2001, 9-36).
} 
For a textbook simple harmonic oscillator, we normally choose these constants so that $\left\langle n\left|H_{B}\right| n\right\rangle=(n+1 / 2) \omega$ for an oscillator of characteristic frequency $\omega$. But this is where our analogy with QFT will begin to appear, if we write the constants in a different way. Consider the energy of a (scalar) bosonic quantum field in one-dimensional spacetime-that is, the energy of a quantum field with a single degree of freedom located at one spatial point, which takes on values at different times $t$. "Particles" are the quanta of excitation of this field. Since there's nowhere for these particles to go in one-dimensional spacetime, their kinetic energy is zero and their only contribution to the total energy of the system is their mass. The field will also have some zero-point energy $E_{0}$. Thus if we label our constants so that

$$
\left\langle n\left|H_{B}\right| n\right\rangle=n m_{B}+E_{0}
$$

where $m_{B}$ is the mass of one particle, the energy eigenstates of our oscillator behave exactly like the energy eigenstates of the one-dimensional bosonic QFT.

This analogy can be taken further. As usual, we can define the lowering operator $a$ and its adjoint, the raising operator $a^{\dagger}$, so that $a^{\dagger}|0\rangle=|1\rangle=a|2\rangle$ and so on. Also as usual, these obey the canonical commutation relations

$$
[a, a]=\left[a^{\dagger}, a^{\dagger}\right]=0 ; \quad\left[a, a^{\dagger}\right]=1
$$

Sticking with our QFT analogy, applying $a^{\dagger}$ to a state adds a particle and applying $a$ subtracts a particle, so we'll call $a^{\dagger}$ the creation operator and $a$ the annihilation operator. These allow us to define a number operator $N_{B}=a^{\dagger} a$, which gives us

$$
\left\langle n\left|N_{B}\right| n\right\rangle=n
$$

so that $H_{B}=N_{B} m_{B}+E_{0}$. Again, the energy is just the mass of the particles plus the zeropoint energy. We can also define a scalar-valued "position" operator. Instead of labeling this $x$ or $q$, we'll call it $\phi(t)$, because it's the analog of the field operator for our one-dimensional scalar field.

These are the relevant features of the bosonic oscillator; except for the analogy with QFT, they will be old hat to anyone who has studied much quantum mechanics. The fermionic oscillator, which is likewise analogous to a one-dimensional free fermionic QFT, will not be 
so familiar.

The crucial difference between the two models arises when we define the creation and annihilation operators for the fermionic oscillator. Instead of obeying the canonical commutation relations (1), the fermion creation operator $c^{\dagger}$ and annihilation operator $c$ will obey the canonical anticommutation relations:

$$
\{c, c\}=\left\{c^{\dagger}, c^{\dagger}\right\}=0 ; \quad\left\{c, c^{\dagger}\right\}=1 .
$$

As a consequence of these relations, $\left(c^{\dagger}\right)^{2}=0$, and thus

$$
c^{\dagger}|1\rangle=\left(c^{\dagger}\right)^{2}|0\rangle=0
$$

This is a simplified case of the Pauli exclusion principle: the oscillator cannot take on energy levels beyond the first. In terms of our QFT analogy, this means that the fermionic field can only contain a single particle.

Similarly to the bosonic case, we can define a fermion number operator $N_{F}=c^{\dagger} c$, with eigenvalues 0 and 1 , and a Hamiltonian $H_{B}=m_{F} N_{F}-E_{0}$. (Interestingly, the ground state energy of the fermionic oscillator is equal but opposite to the ground state energy of the bosonic oscillator (Bagchi, 2001, 12).) Unlike with the bosonic oscillator, there is no scalar position operator. Thinking in terms of the QFT analogy, one would not expect such an operator to exist: the bosonic "position" operator $\phi(t)$ is the analogue of a field operator, and fermionic QFTs cannot have scalar field operators.

Instead, the fermionic oscillator has a Weyl spinor-valued field operator $\chi(t)$, which is the closest analog to a position operator for this system. This can be thought of as a Dirac field with one degree of freedom. (The field's adjoint $\chi^{\dagger}(t)$ is also an important quantity for some purposes.)

\section{$2.1 \quad$ Introducing SUSY}

With both these models on the table, we now have the resources to describe supersymmetry. The SUSY harmonic oscillator is a composite system, formed by taking the tensor product of their state spaces. If $\mathcal{H}_{B}$ is the Hilbert space of states for the bosonic oscillator and $\mathcal{H}_{F}$ is the Hilbert space for the fermionic oscillator, the states of the SUSY oscillator live in the Hilbert 
space $\mathcal{H}=\mathcal{H}_{B} \otimes \mathcal{H}_{F}$. The total Hamiltonian will then be $H=H_{B}+H_{F}=N_{B} m_{B}+N_{F} m_{F}$; the energy is the total mass of the bosons plus the mass of the fermion if one exists.

For this system to exhibit supersymmetry, we must further stipulate that the mass of a boson equals that of a fermion, $m_{B}=m_{F}=m$, which means $H=\left(N_{B}+N_{F}\right) m$. In that case, replacing a boson with a fermion, or vice versa, does not change the total energy. Indeed, we may define operators $Q$ and $Q^{\dagger}$ which do exactly that:

$$
Q=\sqrt{m} a^{\dagger} c
$$

will remove a fermion from the state and add a boson, while

$$
Q^{\dagger}=\sqrt{m} c^{\dagger} a
$$

removes a boson and adds a fermion. ${ }^{3}$ Since these particle-swapping operations don't change the energy, $Q$ and $Q^{\dagger}$ both commute with the Hamiltonian:

$$
[Q, H]=\left[Q^{\dagger}, H\right]=0 .
$$

As a result, $Q$ and $Q^{\dagger}$ generate symmetries-the transformations generated are the SUSY transformations, and $Q, Q^{\dagger}$ are called supercharge operators. This general phenomenon is familiar from many examples, such as the momentum operator acting as the generator of spatial translations, the Hamiltonian as the generator of time translations, or the electric charge as the generator of U(1) gauge transformations. (Because we've introduced only a single independent supercharge operator and its conjugate, we say that this theory exhibits $N=1$ supersymmetry.)

The supercharges don't behave exactly like these other symmetry generators, however. To begin with, because $Q^{\dagger}$ can transform an excited state of a scalar field into an excited state of a spinor field, $Q^{\dagger}$ must itself operate like a spinor (and the same goes for its inverse $Q$ ). This means the $Q$ s must multiply like Grassmann anticommuting numbers. We will discuss Grassmann numbers in more detail soon, but for the moment, this is relevant because it means that the anticommutation relations of the $Q \mathrm{~s}$, rather than their commutation relations,

\footnotetext{
${ }^{3}$ Not that $\left(Q^{\dagger}\right)^{2}=0$, which means that we cannot violate the exclusion principle by replacing multiple bosons with fermions. Only one particle may be swapped. This phenomenon holds generally: SUSY doesn't allow us to create Bose-Einstein condensates out of fermions, for example!
} 
characterize the structure of the symmetries they generate.

Thus, in order to understand the structure of the SUSY transformations, we should look at the anticommutation relations between $Q$ and $Q^{\dagger}$. These are

$$
\{Q, Q\}=\left\{Q^{\dagger}, Q^{\dagger}\right\}=0
$$

and

$$
\left\{Q, Q^{\dagger}\right\}=\sqrt{m} a^{\dagger} c \sqrt{m} c^{\dagger} a+\sqrt{m} c^{\dagger} a \sqrt{m} a^{\dagger} c=m\left(a^{\dagger} a+c^{\dagger} c\right)=m\left(N_{B}+N_{F}\right),
$$

that is:

$$
\left\{Q, Q^{\dagger}\right\}=H \text {. }
$$

Here we've found something surprising: the $Q$ s cannot generate a "group" of symmetries-not actually a group, but a generalized algebraic structure called a supergroup ${ }^{4}$-by themselves. Their anticommutator is the generator of another symmetry-the Hamiltonian $H$, generator of time translations.

This means that the supergroup containing the supersymmetry transformations must also contain time translations. We call it the super-Poincare group; its generators are $Q, Q^{\dagger}$ and $H$. In other words, the supersymmetry transformations cannot be defined on their own within a self-contained algebraic structure. They must be mixed in with spacetime symmetries. (In our toy example of one-dimensional spacetime, the only continuous spacetime symmetry is time translation, but in more realistic versions of SUSY the super-Poincare group contains the whole Poincare group of symmetries of Minkowski spacetime as a subgroup.)

\subsection{Constructing superspace}

This connection with spacetime symmetries suggests that we may be able to understand SUSY as a spacetime symmetry itself. This is the motivation for the superspace formalism. The thought is that instead of existing on the original spacetime that the separate bosonic and fermionic theories were defined on, the SUSY theory can be understood as existing on a different spatiotemporal structure, with the super-Poincare group as the corresponding group of spacetime symmetries. This different spacetime structure is superspace.

\footnotetext{
${ }^{4}$ Roughly, a supergroup behaves like a group except that it includes some elements that multiply like Grassmann numbers.
} 
Superspace is constructed by adding extra dimensions to spacetime-dimensions which are naturally coordinatized by Grassmann anticommuting numbers. These are characterized by the anticommutation relations

$$
\left\{\theta_{i}, \theta_{j}\right\}=\theta_{i} \theta_{j}+\theta_{j} \theta_{i}=0
$$

for any pair of (odd) Grassmann numbers. This means they have some funny properties; for example, it implies that $\theta^{2}=0$ for any Grassmann number $\theta$, which implies (as we noted above) that there are no polynomials over Grassmann variables of order greater than 1 . Grassmann numbers may be combined with ordinary real numbers in a Grassmann algebraa structure that is also sometimes referred to as an algebra of supernumbers, in the context of SUSY. (In such an algebra, the numbers obeying (5) are called "odd" supernumbers, while the other elements are called "even.")

To construct a superspace for our SUSY oscillator, we must add some extra Grassmannvalued coordinates to our representation of spacetime. In this case, a single Grassmannvalued coordinate $\theta$ will do. In our ordinary boson/fermion description of the SUSY oscillator (called the component formulation of the system), we used one-dimensional spacetime with a single coordinate $t$. So our superspace will be two-dimensional, with coordinates $t$ and $\theta$.

Now we need to construct a QFT on our superspace that can be interpreted as physically equivalent to the SUSY oscillator. We define a real scalar superfield $\Phi$ using the boson field $\phi$ and fermion field $\chi$ as inputs:

$$
\Phi(t, \theta)=\phi(t)+i \chi(t) \theta .
$$

This formulation of the superfield allows us to understand the physical meaning of different "locations" along the Grassmann-valued dimension $\theta$. The value of $\Phi(t, \theta)$ at the location $\theta=0$ is just the value of the boson field. At other locations along $\theta$, the fermion field is added to the mix; and $\Phi(t, \theta)-\Phi(t, 0)=i \chi(t) \theta$, which tells us the value of the fermion field at time $t$. So we can think of $\theta=0$ as the "purely bosonic" location in superspace, and the other locations as "mixed boson/fermion" locations.

We may now ask what effect the supercharge operators $Q, Q^{\dagger}$ have on the superfield. Thinking intuitively, given the physical meaning of the superfield operators we just discussed, we would expect the $Q \mathrm{~s}$ to interchange (in some sense) the values of the superfield at the 
bosonic and fermionic locations along $\theta$. Such an interchange is essentially a translation along $\theta$. As we've seen, the square of a SUSY transformation is a time translation, so the supercharges must affect the time coordinate as well. Consider the following transformation on these coordinates:

$$
\theta \rightarrow \theta^{\prime}=\theta+\epsilon, \quad t \rightarrow t^{\prime}=t+i \epsilon \theta
$$

where $\epsilon$ is an infinitesimal Grassmann-valued parameter. This transformation changes the value of the superfield by an amount $\delta \Phi$, where

$$
\delta \Phi=\phi\left(t^{\prime}, \theta^{\prime}\right)-\Phi(t, \theta)
$$

This can be implemented on the state by a generating operator $\mathbf{Q}$, so that $\delta \Phi=\epsilon \mathbf{Q} \Phi$, where

$$
\mathbf{Q}=\frac{\partial}{\partial \theta}+i \theta \frac{\partial}{\partial t}
$$

Note that $\mathbf{Q}^{2}=i \frac{\partial}{\partial t} \Phi$, the time derivative of the field-which is to say, the generator of time translations, or the Hamiltonian! This means that the square of this "superspace translation" generator will generate a time translation, as we expected. In terms of the harmonic oscillator picture of the system, $\mathbf{Q}$ is the "all-purpose" supercharge operator one gets by summing $Q$ and $Q^{\dagger}$,

$$
\mathbf{Q}=Q+Q^{\dagger}
$$

which replaces a fermion with a boson if the state contains a fermion to begin with, and replaces a boson with a fermion otherwise.

The transformations generated by $\mathbf{Q}$ are often called supertranslations, because as we've seen, they translate the state along $\theta$. In SUSY theories on higher-dimensional spacetimes, the same pattern holds, and in general the square of a supertranslation is a spacetime translation. Because $\mathbf{Q}$ is just a more compact way to express the supersymmetry generators, this means that SUSY transformations act like spacetime translations, if we think of superspace as spacetime. This is where our first philosophical puzzle appears: should we think of superspace as spacetime? More generally, how should we interpret this new theoretical structure? 


\section{Three interpretive questions}

In superspace, we have a structure analogous to the spacetime structures described in previous spacetime theories, especially special relativity. Substantivalism-the view that spacetime is an independent substance in some metaphysically robust sense, a "container" for material objects and fields-is a popular interpretation of these theories. We may similarly ask whether superspace is best understood as a substantival container for the fields described by SUSYQFT.

Talk of "containers" is pure metaphor, of course. Before this debate can begin, we must settle on a literal way of stating the superspace substantivalist view in the language of physics and metaphysics. I tend to agree with Jill North (forthcoming), who argues that the disagreement between substantivalists and their relationist opponents should be understood as follows: Substantivalists assert that spacetime structure is a part of the world's fundamental structure, while relationists claim that spatiotemporal facts are metaphysically derivative, depending on (or grounded in) more fundamental facts about material objects and fields. Analogously, we may ask whether superspace is fundamental in SUSYQFT, or whether it is derived from some more basic structure.

Some care is required here, because it is almost certain that our best present-day theories, including supersymmetric quantum field theories, don't really describe the fundamental structure of our world. In particular, spacetime-and superspace-are now thought to be derivative rather than fundamental structures which emerge from some more basic nonspatiotemporal theory of quantum gravity (Huggett and Wüthrich, 2013). So if superspace substantivalism is the thesis that superspace structure is perfectly fundamental, we can be highly confident even before looking at the arguments that superspace substantivalism is false.

To formulate an interesting and potentially true version of superspace substantivalism, while remaining true to the spirit of North's picture, I suggest reformulating the view in terms of relative fundamentality. Some things are more fundamental than others: a quark is more fundamental than a proton, which is more fundamental than a hydrogen atom. Substanti-

valism is (I think) the correct interpretation of general relativity because spacetime is among the most fundamental entities described by the theory, even though general relativity is not a fundamental theory.Similarly, the question of superspace substantivalism should amount 
to the question of whether superspace is among the most fundamental entities described by SUSYQFT. In Section 3.1 below, we will take up this question.

A structure can be (highly) fundamental without qualifying as spacetime structure, and if the prevailing opinion among quantum gravity theorists is correct, spacetime need not be fundamental. We may thus ask a second, logically distinct question about superspace: is it spacetime? That is to say, is it the sort of entity that our concept of spacetime includes, or to which our word 'spacetime' refers? The same question has been asked about other theoretical structures, such as the once-popular electromagnetic ether, the metric in general relativity, and the various possible spacetime structures of Newtonian gravitation theory (Rynasiewicz, 1996; Hoefer, 1998; Knox, 2014). Applied to superspace, we may label this the question of superspace spatiotemporality; Menon argues that superspace ought to count as spacetime on Knox's functionalist approach.

At heart this is a semantic debate, rather than a metaphysical one, although metaphysical questions may need to be answered in order to resolve the debate. Our concept of spacetime may include metaphysical presuppositions about the nature of spacetime, after all. Knox (2014) can be read as suggesting that no metaphysical questions-or at least no difficult onesneed be answered to adjudicate this semantic debate. This strikes me as too hasty, as I will argue in Section 3.2 below. I think the question of superspace substantivalism may need to be answered in order to resolve the debate about superspace spatiotemporality.

A third interpretive question about SUSY concerns the status of bosons and fermions in light of this symmetry. It may seem obvious that SUSY is telling us bosons and fermions are the same sort of entity, and (perhaps more fundamentally) that boson fields and fermion fields are really two ways of describing the same sort of field (the superfield). This is the thesis of ontological unification discussed by Weingard (1988). Weingard argues that ontological unification is only possible if supersymmetry is interpreted as a spacetime symmetry. In my terminology, Weingard argues that superspace spatiotemporality is a necessary condition for ontological unification. In Section 3.3 I'll argue that Weingard is mistaken, and that the superspace anti-realist can also accept ontological unification.

To summarize, we've identified three interpretive puzzles, which concern the status of the following three theses:

Superspace substantivalism: Superspace is among the most fundamental structures described by supersymmetric QFTs. 
Superspace spatiotemporality: Superspace (and not Minkowski spacetime) is the spacetime in which supersymmetric QFTs are set.

Weingard's unification thesis: Superspace spatiotemporality is a necessary condition for the ontological unification of bosons and fermions.

Now it is time to ask what reasons count for and against these interpretive theses.

\subsection{Superspace substantivalism}

We may need an answer to the substantivalism question about superspace in order to issue a verdict about superspace spatiotemporality. Moreover, the substantivalism question is a more substantive question in its own right-a question about the metaphysical interpretation of the theory, not just semantics. But metaphysical questions are also, notoriously, harder to investigate than semantic ones. What sort of purchase can we find on the substantivalism question-and what sort of evidence might bear on it?

The good news is that we have a good sense of which arguments might bear on the general question of substantivalism, from the pre-existing debate in general relativity. The bad news, however, is that superspace substantivalism is not competing with relationism alone. The most interesting rivalry is between the superspace substantivalist and the Minkowski spacetime substantivalist, who asserts that Minkowski spacetime is a more fundamental structure than superspace.

For the sake of clarifying the dialectic between these two varieties of substantivalism, and because I see no new arguments for relationism arising from SUSY, I will assume for the sake of argument that some variety of substantivalism is correct. The question then becomes, what to be a substantivalist about: superspace or Minkowski spacetime? That is to say, on the North (forthcoming) approach I adopt here: is Minkowski spacetime or superspace more fundamental? ${ }^{5}$

A wide variety of theoretical virtues would seem to apply to this question, although their application will be a tricky matter. For example: is Minkowski substantivalism more ontologically parsimonious than superspace substantivalism? The affirmative answer might seem

\footnotetext{
${ }^{5}$ To avoid the misleading appearance of begging the question against either superspace spatiotemporality or its denial, I will do my best to refer to "the thing substantivalists are substantivalist about" not as spacetime, but as the fundamental background structure of a theory.
} 
obvious, since superspace posits additional spacetime structure over and above Minkowski spacetime. But superspace also permits us to make do with fewer fundamental fields, since it allows us to posit a single superfield in place of separate boson and fermion fields. (On the other hand, it isn't obvious that the field operators in QFT should be understood as part of the theory's fundamental structure-see Baker (2009) and the discussion of observables below.) It's impossible to raise all the relevant arguments in a single essay, but I will consider two of the most interesting ones in this section.

Ontological debates are often won or lost on the basis of theoretical parsimony. Where two ontological interpretations of a theory can accomplish the same predictive and explanatory work, we prefer the interpretation that posits fewer entities or types of entity. Let's look at a promising line of argument that seems to suggest that Minkowski substantivalism is preferable to superspace substantivalism in this regard.

To begin, we need an argument for the predictive and explanatory equality of the interpretations. Such an argument is ready to hand. In a mathematically rigorous sense, a superfield theory and its Minkowski-space counterpart describe the same physical quantities (that is to say, the same observables). This equivalence has been proven in simple one-dimensional models like the one presented in Sec. 2 (Hack et al., 2016, 661-662), and is expected to hold in general (Alexander Schenkel, personal correspondence). In essence, the same algebras of observables (and their expectation values as given by the states) can be understood either as assigned to regions of superspace, or as assigned to regions of Minkowski spacetime while exhibiting an additional internal symmetry that relates observables connected by supertranslations.

This equivalence holds only for quantities in the algebras of observables, and so it may seem to be a merely empirical equivalence. But in fact, the algebra of observables in quantum field theory includes every operator that could plausibly be understood to represent a physically significant quantity in the theory's canonical formalism-that is to say, in the absence of an interpretation like Bohmian mechanics that involves non-standard formalism representing ontology (Baker et al., 2015, Section 3).

Arguably, there are some physically significant quantities that don't correspond to operators on the space of states (Wallace, 2008, 21). But note that the plausible candidates for such quantities listed by Wallace are determined by the state's expectation values for observables. In particular, operators outside the algebra of observables generally fail to be 
invariant under permutation, gauge symmetries, or other important symmetries, or fail the condition of relativistic causality. So we have strong grounds for supposing that (within the canonical quantum formalism) theories sharing the same space of states and the same algebra of observables are fully equivalent in explanatory power.

Therefore-somewhat surprisingly-the superspace and Minkowski space versions of a SUSYQFT include the same ontology of physically significant quantities, with the exception of spatiotemporal quantities, which the SUSYQFT includes more of. This may seem impossible. What about the separate boson and fermion fields posited by the ordinary QFT, and the superfields posited by the SUSY theory? The answer is that the equivalence is possible because not all field operators are elements of the observable algebra; only some boson field operators are. So the different-looking fields posited by the two formalisms differ only in terms of operators which are not observables, and which thus lack physical significance. ${ }^{6}$

Bringing this argument to its conclusion: By the above argument for equivalence, the Minkowski and superspace substantivalists can predict and explain all the same experimental phenomena equally well. The Minkowksi substantivalist accomplishes this with a more parsimonious ontology, since Minkowski spacetime has less structure than superspace and the two interpretations otherwise agree on ontology (since they agree about the observables). So Occam's Razor gives us reason to prefer Minkowski substantivalism over superspace substantivalism.

This argument would be decisive, I think, if concrete observable predictions were the only facts supersymmetric QFTs aimed to explain. But on a more ambitious conception of physics-a conception that fits well, in my opinion, with the way high-energy theorists practice their craft-other more general facts also call for an explanation. In particular, we may ask for an explanation of a theory's symmetries in terms of deeper facts, such as the structure of spacetime. In this light, the superspace substantivalist's extra ontology seems more like an asset than a liability. Let me fill in the details of this line of thought with an argument that was suggested to me by James Wells.

Wells's argument for superspace substantivalism proceeds as follows: If we assume Minkowski

\footnotetext{
${ }^{6}$ The two formalisms may still differ about which observable operators signify fundamental quantities, as opposed to merely physically significant (but derivative) quantities. I wish I had room in the present paper to explore the implications of this possible disagreement, but it is worth noting that it may imply further differences in explanatory power between the Minkowksi substantivalist and superspace substantivalist ontologies, beyond the ones discussed here.
} 
structure is more fundamental than superspace structure, this leaves us with a sort of finetuning puzzle. Since the most fundamental background structure is Minkowski spacetime, this structure could support a variety of non-supersymmetric laws for the same fields. In particular, the masses of the boson and fermion fields need not have been equal. We are left with no explanation for why SUSY is a symmetry at all-the equality of the masses must be left unexplained, a brute posit.

If superspace is the more fundamental structure, on the other hand, and the system is most fundamentally described as a superfield, there is a ready explanation for the equality of the masses. For the only possible superfield theories that can be formulated on superspace exhibit equal masses for the (less fundamental) boson and fermion fields.

Wells's argument depends on the assumption that fundamental spacetime structure can explain the dynamical symmetries of basic physical laws. This assumption has been questioned by Brown (2005), who argues that in general, good explanations will go in the other direction-the symmetries of the dynamics explain the symmetries of spacetime, not vice versa. We must, Brown suggests, "consider absolute space-time structure as a codification of certain key aspects of the behavior of particles" (Brown, 2005, 24). This view implies, however, that the behavior of particles is more fundamental than spacetime structure-a claim that is incompatible with substantivalism defined in North's terms.

While the substantivalist cannot accept Brown's own view, Brown is right to insist that there is no impossibility (logical or metaphysical) of disagreement between the symmetries of spacetime and the symmetries of the laws. But I agree with Skow (2006) and Janssen (2009), who suggest that there may be a higher-order law of nature requiring dynamical symmetries to mirror the symmetries of the fundamental background (spacetime) structure. ${ }^{7}$

At any rate, it seems obvious to me that explaining dynamical symmetries in terms of the symmetries of fundamental background structure is a justified method of theorizing in physics. This allows us to escape the primary pitfall of Brown's dynamical picture, namely the glaring lack of any explanation for the coincidence-highly surprising, on Brown's picture-

\footnotetext{
${ }^{7}$ Such a law will have to relate a theory's dynamical symmetries to its absolute background geometrical structure, of course. In a theory like general relativity, for example, which admits of many solutions with different spacetime structures, we should not require that the symmetries of the force laws vary from solution to solution. But a similar law in general relativity could explain the local Lorentz covariance of the laws of general relativistic field theory, since Lorentz covariance is a local symmetry of all general relativistic spacetimes.
} 
that all of the different relativistic matter fields share the same (local) external symmetries, and that these match the symmetries of the metric. On Brown's picture, no explanation is possible for the fact that we don't live in a world where some fields exhibit Galilean invariance while other fields exhibit Lorentz invariance. Since the dynamical laws governing the different fields are explanatorily prior to spacetime structure, it is simply a coincidence that those dynamical laws all happen to agree on their external symmetries. In later work, Brown and co-authors refer to this coincidence as a "miracle" of relativity (Read et al., preprint, 7).

But if it is a law that the symmetries of the matter fields must match those of the spacetime (fundamental background) metric, this fact is no coincidence and no miracle. And if we assume such a law, Wells's argument for superspace substantivalism is compelling. Indeed, the manifest persuasiveness of Wells's argument for superspace substantivalism further underscores the implausibility of Brown's picture of spacetime. We have here a good argument in favor of superspace substantivalism. It is by no means a decisive argument, and there is much more to be said on both sides of this interpretive question, but considerations of space compel me to move on to the question of spatiotemporality.

\subsection{Superspace spatiotemporality}

Menon (forthcoming) is primarily concerned with the question of superspace spatiotemporality: whether superspace deserves the name 'spacetime.' (To avoid endless repetition of an awkward phrase, let's shorten the view's name to 'spatiotemporality.') His answer to the spatiotemporality question is yes (insofar as superspace fills the role he calls theoretical spacetime) and no (insofar as it fails his criteria to count as operational spacetime).

One of the theoretical roles of spacetime structure is to connect the symmetries of different matter fields-e.g., the Lorentz invariance of relativistic spacetimes extends to the Lorentz invariance of matter fields defined on such spacetimes. This is the role Menon identifies with theoretical spacetime. He initially defines theoretical spacetime as "That structure which

picks out the (local) symmetries of the dynamical equations governing matter fields" (Menon, forthcoming, 3). But as he recognizes, this first-pass definition requires further precisification to accommodate the fact that we don't ordinarily treat spacetime as determining all the symmetries of matter fields. Different matter fields with different symmetries can be defined 
(or even coexist) on the same spacetime; for example, the quark and gluon fields possess an $S U(3)$ gauge symmetry that the electromagnetic field lacks, even though all these fields are defined on Minkowski spacetime.

So Menon further qualifies his definition in a natural way: theoretical spacetime picks out only the external symmetries of the matter fields. 'External symmetry' is often treated as another term for spacetime symmetries, so to avoid the risk of circularity, Menon stipulates his own definition: an external symmetry is a symmetry shared (in a sense I will explain in a moment) by all the different matter fields (Menon, forthcoming, 5). So theoretical spacetime is the structure required to specify the family of external symmetries shared by all matter. As Menon notes, this accords with Earman's well-known postulate that "Any dynamical symmetry of [a theory] $T$ is a spacetime symmetry of $T$ " and vice versa (Earman, 1989, 46).

Beginning from Menon's definition, it is straightforward to show that superspace is theoretical spacetime in SUSY-invariant theories. To identify the external symmetries, Menon begins with the parameter space expression for a field theory, in terms of n-tuples of real numbers specifying the theory's states. For example, a four-dimensional real scalar field theory's parameter space (in this sense) will be given by quintuples (the four spacetime dimensions for a point plus the field value at that point), while a complex field on the same spacetime will be given by sextuples (since it takes two real numbers to specify a complexvalued field at a point). In special relativity, assuming the dynamics is independent of the complex field's phase, the complex field's parameter space can be thought of as breaking down into two parts: one invariant under $U(1)$, and one invariant under the Poincare group. The real field will also have a corresponding section of its parameter space which is invariant under the Poincare group. For Menon, this is how we know that the Poincare group gives the external symmetries of special relativistic field theories. The external symmetry group, on his definition, is the symmetry group of the common parameter space of all the fields.

In a SUSY theory defined on superspace, the super-Poincare group will be the largest invariance group of the fields' common parameter space. So Menon's definition will identify the super-Poincare group as the external symmetry group, and superspace as theoretical spacetime.

Clearly a lot is built into Menon's definition of theoretical spacetime, since the argument for spatiotemporality is extremely straightforward once we accept his definition. We must ask whether the definition captures what we are after when we raise the question of spa- 
tiotemporality. Since this is ultimately a semantic question-a question of how best to apply our pre-existing spacetime concept-the best way to proceed is by considering cases.

Menon openly admits one counterintuitive consequence of his definition: when dealing with examples of a single field on spacetime, "there is no principled reason to refer to one parameter space rather than the other as the external space" (Menon, forthcoming, 5). For example, consider an example like the one above, but minus the real scalar field-that is, a special relativistic world with only a complex scalar field exhibiting $U(1)$ symmetry. This theory's parameter space splits into a $U(1)$-invariant part and a Poincare-invariant part. Both of these symmetry groups are symmetries of all the matter fields, since there is only one field. So while Menon's definition tells us that only one of these two groups, $U(1)$ or the Poincare group, represents the external symmetries, the definition cannot tell us which one is the external symmetry group. And since he defines theoretical spacetime as the geometric structure needed to pick out the external symmetries, there is no fact of the matter in this example whether that structure is Minkowski space (the geometry characterized by Poincare symmetry) or the complex unit circle (the geometry characterized by $U(1)$ symmetry).

This ambiguity does not only arise in the case of a single field. Consider the example of quantum electrodynamics (QED). QED describes two quantum fields, an electron (Dirac) field and a photon (Maxwell) field. But these two fields as a composite system are jointly invariant under a local $U(1)$ gauge symmetry, which transforms both the photon field's potential and the electron field's phase. As a result, there will similarly be no way for Menon's definition to determine whether it is the Poincare symmetry of QED or its local gauge symmetry which represents its external symmetry-even though common sense dictates that the theory's Poincare invariance, and not its gauge invariance, represents the symmetries of spacetime.

How serious of a problem is all this for Menon's definition of theoretical spacetime? Given his aim of advancing a definition that can determine whether a new and unfamiliar geometric structure, superspace, falls under our existing spatiotemporal concept, I think the problem is quite serious. I grant that Menon's definition will give the correct result about the spacetime structure of physically realistic ordinary field theories like the Standard Model, which describe more then one interaction. But first, one might worry that even more realistic future field theories might pursue the unification of the forces ("grand unification") in such a way as to conjoin their internal symmetries, thus giving rise to the same problem that appears 
for Menon's definition in the case of QED. And second, when applying Menon's definition of theoretical spacetime to the conceptually ill-understood case of SUSY, it is difficult to be confident about its verdict given that the definition's extension does not unambiguously include some paradigm cases of spacetime in field theories such as QED.

There is a further, and potentially more troubling, problem for the full generality of Menon's definition of theoretical spacetime: His definition offers no guarantee that a physically intelligible geometric structure invariant under the external symmetries (as he defines them) will always exist. Supersymmetry is actually an interesting test case here, since for some complex SUSY theories it is not yet known whether a superspace formalism exists (Castellani et al., 2014, 420). For example, $N=4, D=4$ supersymmetric Yang-Mills theory (a gauge theory on four-dimensional spacetime with four independent supercharge operators) no superfield formulation of the theory has yet been constructed. This is not a problem that arises for the minimal supersymmetric Standard Model, the most physically realistic SUSYQFT. But it is concerning to say the least that it remains an open question, for some SUSY theories, whether any geometric structure meeting Menon's definition of theoretical spacetime even exists. Surely if it is proven that no superspace version of $N=4$ superYang-Mills theory exists, we should not conclude that this theory is not set on (theoretical) spacetime. Rather, the natural conclusion would be that the theoretical spacetime setting of the theory is Minkowski spacetime.

Getting ahead of myself for a moment, I think these problems arise from Menon's presuming to offer necessary and sufficient conditions for a concept ("spacetime") that I take to have the more complicated structure of a cluster concept. To make this point of disagreement clearer, I will first consider Menon's other argument for spatiotemporality before presenting my own view.

In addition to his theoretical spacetime definition, Menon considers Eleanor Knox's inertial functionalist account of spacetime. Knox's view is functionalist in the sense that it identifies spacetime as whatever geometric structure plays a particular role in the laws, which she calls its functional role, on analogy with functionalism about the mind. Menon's symmetry-based definition of theoretical spacetime is also a functionalist definition in this sense. But instead of requiring, as Menon does, that spacetime determine the external symmetries, Knox's functionalism holds that spacetime is the geometric structure required to 
determine inertial frames. ${ }^{8}$

Menon argues that Knox's inertial functionalist picture gives the same answer about spatiotemporality as his own definition of theoretical spacetime: superspace is spacetime, on Knox's picture. I disagree, though, with Menon's application of Knox's view to SUSY. It seems to me that Knox's view lacks the resources to tell whether superspace or Minkowski spacetime is spacetime. To show why, let me first explain how Menon employs her definition.

For Knox, spacetime is the (minimal) theoretical structure required to determine the inertial frames for a theory. Frames for Knox are coordinate systems in which the theory can be expressed. The inertial frames are then the coordinate systems meeting these criteria:

1. Inertial frames are frames with respect to which force free bodies move with constant velocities.

2. The laws of physics take the same form (a particularly simple one) in all inertial frames.

3. All bodies and physical laws pick out the same equivalence class of inertial frames (universality). (Knox, 2013, 348)

The first criterion may not apply in non-mechanical theories with no ready definition of force or velocity, in which case Knox would call a structure spatiotemporal if it satisfies the other two criteria. Unless we want to beg the question against superspace spatiotemporality, we must not apply criterion 1 to SUSY, since it isn't clear what it would mean for a force or velocity vector to point along the Grassmann-valued directions.

What about Knox's criteria 2 and 3? As Menon points out, superspace does possess its own analogue of inertial frames-coordinate systems sometimes called "superinertial frames," in which the superfield and the dynamical equations governing it take on their simplest form. In these coordinate systems, the formula for the superspace anologue of the spacetime interval also takes on its simplest form; additionally, the superinertial frames are related by super-Poincare transformations (Buchbinder and Kuzenko, 1998, 160). Menon infers that, applying Knox's criteria to the theory of a superfield, we must conclude that the coordinate systems meeting criteria 2 and 3 are the superinertial frames. Therefore, since superspace is

\footnotetext{
${ }^{8}$ Knox's account actually focuses on local inertial frames, but since the scope of this paper only includes flat spacetimes, I'll follow Menon in leaving that complication aside and considering only the special case of global inertial frames.
} 
the structure required to define superinertial frames, superspace must be spacetime in SUSY theories.

The problem with Menon's reasoning here is that the same line of inference gives a different answer if we apply Knox's criteria to a SUSY theory written in its component form (in terms of separate Bose and Fermi fields) rather than its superfield form. In that case, the relevant coordinate systems will be ordinary Minkowski coordinate systems, and the ones giving the simplest expressions of the (component) fields and their dynamics will be the inertial frames of ordinary Minkowski spacetime, not the superinertial frames. By applying Knox's criteria to the superfield form of the theory rather than its component form, Menon effectively treats the coordinate systems on superspace as the relevant coordinate systems to which we should apply Knox's criteria. He has not really given an argument from Knox's picture to superspace spatiotemporality. Instead he has given us an argument from Knox's picture, plus the assumption that the superfield form of a SUSY theory is its proper formbut this begs the question against the denier of spatiotemporality, who will surely insist that the component form of the theory is more basic.

This underscores a weakness of Knox's inertial definition of spacetime. The case of supersymmetry illustrates that Knox's definition does not (as one might hope) take as input a theory and return an answer about which of that theory's structures represent spacetime. Instead, it takes as input a theory plus a class of descriptions of that theory in different coordinate systems; only with all that information can Knox's definition be applied to give an answer about which of the theory's structures are spatiotemporal.

This aspect of her definition alone should be disturbing to those of us who believe that the real physical content of a theory, including what it tells us about spacetime, should be a coordinate-independent matter. But even worse, as the case of SUSY also illustrates, it sometimes matters which class of coordinate systems one provides as input to Knox's definition. Applying Knox's criteria to the class of Minkowski coordinate systems, her criteria give the answer that Minkowski spacetime is spacetime. Feeding in the superspace coordinate systems, on the other hand, gives an entirely different answer.

I have argued elsewhere Baker (preprint) that there are several other reasons to reject Knox's inertial version of spacetime functionalism, even assuming (as seems plausible) the correctness of the broader functionalist picture that questions about spatiotemporality should be answered by looking at each theoretical structure's role in a theory's laws. (In brief, Knox's 
version of functionalism cannot be applied to theories with no inertial structure or local degrees of freedom, such as topological quantum field theories, even though such theories may possess many other attributes we ordinarily think of as spatiotemporal.) Instead, we should think of our spacetime concept as a cluster concept (Baker, preprint, Section 4).

On this cluster concept picture, there is no one simple set of necessary and sufficient conditions for an entity in our theories to count as 'spacetime.' Rather, there are many independent criteria for falling under that concept. To apply it, we must look at a theory to see whether one or more structures meet a sufficient number of these criteria. If there is a sufficiently satisfactory realizer of the concept in our theory, then either we may count each sufficiently good realizer as one variety of spacetime described in that theory, or else count only the best realizer as spacetime. (This choice point gives rise to two different versions of the cluster concept picture, one on which there must be a single unique spacetime in each theory, and another on which multiple classes of structures may qualify as spatiotemporal.)

If we choose the more permissive version of the cluster view, on which a single theory may describe "multiple spacetimes," it seems likely that both Minkowski spacetime and superspace should qualify as spatiotemporal. Minkowski spacetime is obviously a strong enough candidate to count as spacetime, since that is the role it plays in ordinary special relativity. And the reasons Menon adduces in favor of superspace spatiotemporality are certainly compelling: superspace has a central role in codifying the symmetries of SUSY theories and its analogues of familiar spatiotemporal features like the interval and inertial frames are highly suggestive.

What if we assume, on the other hand, that there can be only one spacetime? One might assume, as I argue in Baker (preprint, Section 5), that the more fundamental structure is a better realizer for the spacetime concept, other things being equal. If that is indeed a significant criterion for the spacetime cluster concept, the arguments of the previous section count in favor of superspace spatiotemporality as well as superspace substantivalism. On the other hand, superspace fails many criteria that we ordinarily think of when we imagine spacetime. As Menon points out, in his argument that superspace is not "operational spacetime," there are no clear analogues of rods and clocks that measure the metric on superspace in the same way that ordinary (ideal) rods and clocks measure the Minkowski metric in special relativity (Menon, forthcoming, Section 4.2). In many ways, the Grassmann-valued dimensions do not behave like spatial dimensions. Depending on how heavily we weight the importance 
of these operational characteristics, as opposed to fundamentality, either conclusion on the spatiotemporality question could conceivably be justified. This amounts to a question of conceptual analysis, or of the semantics of our theoretical term 'spacetime,' and borderline questions of this sort can be difficult to resolve. A systematic survey of competent users (physicists?) might be required.

Let me close this section with an observation suggested to me by Tim Maudlin. It may be that a satisfactory interpretation of supersymmetry requires revision or (depending on how one looks at it) replacement of our existing spatiotemporal concepts. When Minkowski (1909) proposed that we conceive of ourselves as existing in a four-dimensional geometric structure rather than a three-dimensional one, his recommendation was not that we subsume this notion of spacetime under our existing concept of space. Rather, the idea that came to define so much of subsequent physics, and its interpretation, posited an altogether new sort of entity. Our understanding of this entity has been illuminated by the ways it is analogous to our pre-existing spatial concept. But it would be wrong-a misuse of words-to suggest that spacetime is simply another variety of space.

To suggest that superspace is another variety of spacetime may involve a similar error. There are many analogies between superspace and the sort of structure we ordinarily identify with spacetime; also many disanalogies. Perhaps what is needed (and underway in the theory of SUSY) is the introduction of a new concept. It could even be that something akin to Menon's notion of theoretical spacetime, as opposed to spacetime simpliciter, is the new concept we need (although for the reasons cited above, I don't think Menon's precise definition of theoretical spacetime will do). Since these are semantic questions, and the use of the relevant terms ('spacetime,' 'superspace') and concepts is currently in flux, there may be no fact of the matter as yet. I think this suggests that the most interesting question in the vicinity is not actually the semantic/conceptual question, but the substantive metaphysical question about substantivalism discussed in the previous section. But for the reader interested in how best to subsume SUSY under our existing spatiotemporal concepts, I offer the arguments of this section as my best attempt. 


\subsection{Weingard's unification thesis}

One way of posing the question of superspace spatiotemporality is to ask whether SUSY is a spacetime symmetry (like time reversal, rotations and boosts) or an internal symmetry (analogous to phase transformations in quantum mechanics). Weingard has suggested that a lot hinges on this question-not just the interpretation of superspace, but also our naive understanding of bosons and fermions as separate species of particle.

Consider the photon and its supersymmetric partner, the photino. In a supersymmetric world, it seems natural to suggest that these are really two states of a single species of particle. Perhaps the difference between a photon and a photino is analogous to the difference between a spin-up electron and a spin-down electron. Weingard argues that this is the right way to understand SUSY-as an ontological unification of bosons and fermions-but only if SUSY is a spacetime symmetry.

Weingard suggests-rightly, in my opinion (Baker, 2011) - that states related by a spacetime symmetry are physically equivalent even if that symmetry is spontaneously broken (as SUSY is likely to be if it is a symmetry of our world). "[T]he difference between a spin $z=1 / 2$ electron and $z=-1 / 2$ electron is that of the orientation of a single particle. And this is so even if the symmetry is broken by a magnetic field and the two have different energies" (Weingard, 1988, 103). But the same does not hold, according to Weingard, for internal symmetries.

He considers the case of isospin, an approximate symmetry that transforms protons into neutrons (by transforming up quarks into down quarks). As a matter of group theory, this symmetry has an $S U(2)$ structure isomorphic to the structure of rotations of electrons-the up quark is treated as the "isospin-up" state and the down quark as the "isospin-down" state. But we are not tempted in this case to say that these quark varieties are different states of the same particle type.

The reason we are not tempted, Weingard claims, is that "the symmetry transformations are merely 'rotations' in an internal space like isospin space. Because such an internal space is, apparently, just a mathematical space, the symmetry transformations just represent the fact that the particles can be transformed into each other" (Weingard, 1988, 103). In this case the symmetry does not signify physical equivalence of states, but rather the dynamical possibility of one state evolving into another. Generalizing from this example, Weingard concludes that SUSY will only imply the physical equivalence of bosons and fermions if it is 
a spacetime symmetry.

I don't accept Weingard's reasoning here, however. Many times in physics, rotations within an internal space $d o$ imply physical equivalence between the states related by these rotations. The most obvious example is phase transformations in quantum mechanics: to multiply the global state by a phase is to rotate the state vector within an internal space. But (almost) no one denies that global states related by a phase are the same state physically.

As Weingard observes, isospin rotations aren't a reason to consider up and down quarks to be two states of the same fundamental particle type. But this is not because isospin is an internal symmetry. It's because isospin is only an approximate symmetry, at least within the Standard Model-so fundamentally speaking, it isn't a symmetry at all. The fundamental interactions of the theory (given by the Standard Model Lagrangian) recognize the difference between these species of quark, assigning them different fundamental masses and charges. Isospin is not a spontaneously broken symmetry (as SUSY is conjectured to be)-it is, as they say, broken by the Lagrangian.

We may discover in the future that the Standard Model is inaccurate in this regard, and the differences between up and down quarks are the result of spontaneous symmetry breaking. In that case, I think we should conclude that these different species of quark really are different states of the same type of particle, even though the symmetry that relates them would be an internal symmetry.

In short, Weingard's argument fails because he assumes that internal symmetries cannot ground physical equivalence of states as spacetime symmetries do. But they can-and so there is no obstacle to the ontological unification of ordinary particles with their supersymmetric partners, even if SUSY is not understood as a spacetime symmetry. If SUSY is a fundamental symmetry, the photon and the photino are best understood as two states of the same elementary particle type.

\section{Conclusions}

Of the interpretive questions raised here, the only one I take to be closed is Weingard's unification question. Although I have concerns about its ultimate interest as a philosophical puzzle as opposed to a purely semantic one, the question of spatiotemporality has thus far only been addressed from perspectives, like mine and Menon's, which are broadly sympa- 
thetic to functionalism about spacetime. On alternative views holding that spacetime has some metaphysically robust essence, the question may prove more substantive.

The question of superspace substantivalism is clearly substantive, and although I have argued in its favor, there remain many further angles to be considered. One example: suppose it turns out that the nonexistence of a superspace formalism can be rigorously proved for some non-realistic but conceptually interesting SUSY theories. How should this affect our opinion of whether superspace is fundamental in those theories where it does exist?

There also remain many questions aside from these which have not been addressed anywhere in the literature. Many of these will require more complicated physics than the toy model presented in this paper. For example, I've considered only "rigid" supersymmetry (supersymmetry which acts as a global symmetry). How do the puzzles raised here change in the context of supersymmetry which acts non-rigidly, on analogy with a local gauge symmetry? How should our interpretation of SUSY change in the context of superstring theory? Lastly, I discussed in Section 3.1 the open question of how to understand the equivalence between component and superspace formulations of the same SUSY theory. Given the fervid recent interest in theoretical equivalence among philosophers of physics (e.g. Coffey, 2014; Rosenstock et al., 2015; Barrett and Halvorson, 2016), this could prove to be a highly illuminating test case.

Although the future of supersymmetry as a theory of our universe is uncertain, I see a bright future for the foundational interpretation of supersymmetry. It is too puzzling and fruitful a topic to be ignored any longer.

\section{Acknowledgements}

Thanks to Tim Maudlin and James Wells for discussion that inspired some of the arguments presented here, and to Tushar Menon for many months of edifying discussion, correspondence and comments.

\section{References}

Bagchi, Bijan Kumar (2001), Supersymmetry in Quantum and Classical Mechanics, New York: Chapman and Hall/CRC. 
Baker, David John (2009), "Against Field Interpretations of Quantum Field Theory," British Journal for the Philosophy of Science 60:585-609.

Baker, David John (2011), "Broken Symmetry and Spacetime," Philosophy of Science 78:128-148.

Baker, David John (preprint), "On Spacetime Functionalism," .

Baker, David John, Hans Halvorson and Noel Swanson (2015), "The Conventionality of Parastatistics," The British Journal for the Philosophy of Science 66:929-976.

Barrett, Thomas William and Hans Halvorson (2016), "Glymour and Quine on Theoretical Equivalence," Journal of Philosophical Logic 45:467-483.

Brown, Harvey R. (2005), Physical Relativity: Space-time Structure from a Dynamical Perspective, Oxford: Oxford UP.

Buchbinder, Ioseph L. and Sergei M. Kuzenko (1998), Ideas and Methods of Supersymmetry and Supergravity, London: IOP Publishing.

Castellani, L., R. Catenacci and P.A. Grassi (2014), "Supergravity actions with integral forms," Nuclear Physics B 889:419 - 442.

Coffey, Kevin (2014), "Theoretical Equivalence as Interpretative Equivalence," British Journal for the Philosophy of Science 65:821-844.

Earman, John (1989), World Enough and Spacetime, MIT press.

Hack, Thomas-Paul, Florian Hanisch and Alexander Schenkel (2016), "Supergeometry in Locally Covariant Quantum Field Theory," Communications in Mathematical Physics 342:615-673.

Hoefer, Carl (1998), “Absolute Versus Relational Spacetime: For Better or Worse, the Debate Goes On," British Journal for the Philosophy of Science 49:451-467.

Huggett, Nick and Christian Wüthrich (2013), "Emergent spacetime and empirical (in)coherence," Studies in History and Philosophy of Modern Physics 44:276-285.

Janssen, Michel (2009), "Drawing the Line Between Kinematics and Dynamics in Special Relativity," Studies in History and Philosophy of Modern Physics 40:26-52.

Knox, Eleanor (2013), "Effective Spacetime Geometry," Studies in History and Philosophy of Modern Physics 44:346-356.

Knox, Eleanor (2014), "Newtonian Spacetime Structure in Light of the Equivalence Principle," British Journal for the Philosophy of Science 65:863-880. 
Menon, Tushar (forthcoming), "Taking up superspace - what would it take to be a realist about superspace?" in Huggett, Nick, Baptiste Le Bihan and Christian Wüthrich (eds.), Philosophy Beyond Spacetime: The Philosophical Foundations of Spacetime, Oxford: Oxford UP.

Minkowski, Hermann (1909), "Space and time," Annual Report of the German Mathematician Association 18:75-88.

North, Jill (forthcoming), "A New Approach to the Relational-Substantival Debate," Oxford Studies in Metaphysics .

Read, James, Harvey R. Brown and Dennis Lehmkuhl (preprint), "Two Miracles of General Relativity," .

Rosenstock, Sarita, Thomas William Barrett and James Owen Weatherall (2015), "On Einstein Algebras and Relativistic Spacetimes," Studies in History and Philosophy of Science Part B: Studies in History and Philosophy of Modern Physics 52:309-316.

Rynasiewicz, Robert (1996), “Absolute Versus Relational Space-Time: An Outmoded Debate?" Journal of Philosophy 93:279-306.

Skow, Bradford (2006), "Review of Harvey R. Brown, Physical Relativity: Space-Time Structure From a Dynamical Perspective," Notre Dame Philosophical Reviews 2006.

Susskind, Leonard (2012), "Supersymmetry and Grand Unification," Stanford University lecture series (accessed April 27, 2017).

URL https://www .youtube.com/playlist?list=PL_IkS0viawhpBbVkmwRLiysdV5eXegvd_

Wallace, David (2008), "Philosophy of Quantum Mechanics," in Rickles, Dean (ed.), The Ashgate Companion to Contemporary Philosophy of Physics, Burlington, VT: Ashgate, 16-98.

Weingard, Robert (1988), "A Philosopher Looks at String Theory," PSA: Proceedings of the Biennial Meeting of the Philosophy of Science Association 1988:95-106. 